\title{
Composición nutricional del Tripsacum laxum fertilizado con nitrógeno, fósforo y potasio ${ }^{1}$
}

\section{Nutritional composition of Tripsacum laxum fertilized with nitrogen, phosphorous and potassium}

\author{
Carlos Boschini-Figueroa ${ }^{2}$, Claudio Fabián Vargas-Rodríguez ${ }^{2}$
}

\begin{abstract}
Resumen
El conocimiento de las prácticas culturales que permiten obtener el mayor beneficio del potencial de las fuentes forrajeras es necesario para el éxito en la producción animal. El objetivo de este estudio fue explorar la respuesta bromatológica del Tripsacum laxum a la fertilización con diferentes niveles de nitrógeno, fósforo y potasio aplicado al suelo. La investigación se realizó en la Estación Experimental de Ganado Lechero "Alfredo Volio Mata" de la Universidad de Costa Rica, localizada en Ochomogo, Cartago, Costa Rica. Se utilizó un diseño de bloques al azar con cuatro repeticiones y los tratamientos dispuestos en un arreglo factorial de compuesto central y rotable 53. La materia seca (MS), proteína cruda (PC), fibra neutro detergente (FND) y el extracto etéreo (EE) fueron determinados. Dependiendo de los niveles de fertilización con N, P y K se obtuvieron las dosis apropiadas para lograr contenidos de nutrientes máximos en cada uno de los parámetros bromatológicos evaluados en este estudio, y fue posible optimizar la composición de la mezcla fertilizante según el componente nutricional que se quiera privilegiar en particular ya sea en la hoja, en el tallo o en la planta entera. No existe una dosis de fertilizante única para mejorar todos los componentes nutricionales de la planta.
\end{abstract}

Palabras clave: forrajes, relaciones planta suelo, respuesta de la planta, plantas forrajeras.

\begin{abstract}
Getting to know the knowledge that cultural practices have allows researchers to obtain the greatest benefit from the potential of fodder sources, this knowledge is necessary for animal production success. The aim of this study was to explore the bromatological response of Tripsacum laxum to fertilization with different levels of nitrogen, phosphorus, and potassium that were applied to the ground. The investigation was conducted at the "Alfredo Volio Mata" Experimental Dairy Farm a Dairy farm that is part of the University of Costa Rica and that is located in Ochomogo, Cartago, Costa Rica. In the investigation a randomized block design with four replications and treatments arranged in a factorial central composite and on a rotatable 53 was used. Dry matter, crude protein, neutral detergent fiber, and ether extract were determined. Depending on the levels of fertilization with $\mathrm{N}$, P, and $\mathrm{K}$, the appropriate doses were obtained in order to reach the maximum nutrient contents in each of the bromatological parameters evaluated in this study, it was possible to optimize the composition of the fertilizer mixture based on the nutritional component to be particularly favored, either on the leaf, on the stem, or on the whole plant. No single fertilizer dose was able to improve all nutritional components of the plant.
\end{abstract}

Keywords: forage, plant soil relation, plant response, feed crops.

1 Recibido: 22 de febrero, 2017. Aceptado: 3 de abril, 2017. Este trabajo formó parte del proyecto de investigación inscrito en la Vicerrectoría de Investigación, No 737-97- 006. Universidad de Costa Rica, San José, Costa Rica.

2 Universidad de Costa Rica, Facultad de Ciencias Agroalimentarias, Estación Experimental Alfredo Volio Mata. carlos.boschini@ucr.ac.cr, fabian.vargas@ucr.ac.cr 


\section{Introducción}

El desarrollo de explotaciones ganaderas ha generado una alta demanda de materiales forrajeros de buena calidad (Suyama et al., 2007), esto ha ocasionado que los productores se vean obligados a utilizar una serie de insumos que suplan a los pastos la cantidad de nutrientes necesarios para soportar un manejo intensivo de cosecha (Cherney et al., 1994). Sin embargo, los cultivos forrajeros han sido los que menos atención han recibido en materia de fertilización o se han sostenido bajo esquemas muy pobres que no suplen por completo las necesidades de producción (Cástino, 2007).

La mayoría de las fórmulas químicas se enfocan en fuentes nitrogenadas fosfóricas y potásicas, debido a que, son los elementos que más limitan el crecimiento y la composición nutricional de los forrajes en general (Torres y Lemos, 2009). El fósforo cuando es deficiente reduce la expansión foliar, lo cual ocasiona un amarillamiento y senescencia prematura de las hojas maduras, el crecimiento aéreo se reduce y ocasiona que una mayor proporción de nutrientes disponibles en la planta se destine al desarrollo radicular, lo que provoca una subutilización de recursos que se traduce en producciones pobres de forraje (Quintero et al., 1997). Forma parte de los compuestos orgánicos ricos en energía y es indispensable en procesos como la síntesis de proteínas, grasas y almidones en las plantas (Romero y Márquez, 2002; Mehrvarz y Chaichi, 2008).

La carencia de nitrógeno afecta la acción fotosintética, debido a una reducción en la capacidad de uso del carbono. Esto implica una merma en la actividad metabólica de los cloroplastos y por consiguiente, una menor presencia de nitrógeno en los tejidos de asimilación (Veneciano y Frigerio, 2008).

El potasio está muy relacionado con la capacidad de intercambio de cationes, su deficiencia altera la disponibilidad de otros elementos, además tiene una importante participación en el metabolismo de los ácidos nucleicos, proteínas, vitaminas y las sustancias de crecimiento (Bednarz y Oosterhuis, 1999).

La carencia de los elementos mayores afectan a los forrajes como el pasto Prodigio, Prodigioso o Guatemala (Tripsacum laxum), conocido también como Tripsacum fasciculatum y Tripsacum andersonii (Chen, 1992), que es utilizado bajo sistemas muy intensivos, con frecuencias de cosechas cortas, donde la demanda de los nutrientes es alta y la velocidad de crecimiento está ligada a los elementos que el suelo pueda aportar, limitación que se manifiesta en pobres rendimientos y contenido nutricional (Bertsch, 1998). Este material, ubicado en la misma línea filogenética del teosinte, es un pariente silvestre del maíz (Zea mays). El género Tripsacum es el único con el cual se ha podido cruzar el maíz en condiciones experimentales y se han producido híbridos viables que crecen hasta alcanzar la madurez (Leblanc et al., 1995).

Actualmente hay muy poca información con respecto al efecto del nitrógeno, fósforo y potasio sobre la calidad del T. laxum. Existe entonces la necesidad de implementar programas de fertilización precisos para el cultivo y que no causen daños al ambiente.

Con esa base, se ejecutó el presente trabajo cuyo objetivo fue explorar la respuesta bromatológica del Tripsacum laxum a la fertilización con diferentes niveles de nitrógeno, fósforo y potasio aplicado al suelo.

\section{Materiales y métodos}

\section{Localización}

El experimentó se ejecutó en la Estación Experimental de Ganado Lechero Alfredo Volio Mata de la Universidad de Costa Rica, durante el último trimestre del año 2013. Esta unidad está localizada a $1542 \mathrm{msnm}$ en Ochomogo de Cartago, donde la precipitación media anual es de $1502 \mathrm{~mm}$ (concentrada en la época de mayo a noviembre). La humedad relativa media es de $88 \%$ y la temperatura promedio anual de $17,9{ }^{\circ} \mathrm{C}$ (IMN, 2010). El ecosistema 
de la zona es considerado como bosque húmedo montano bajo (Vásquez, 1982). El suelo es de origen volcánico, clasificado como “Typic Hapludands”, presenta buen drenaje y una fertilidad media (Soil Survey Staff, 2014).

\section{Procedimiento experimental}

El área empleada para establecer el cultivo fue de $5000 \mathrm{~m}^{2}$ aproximadamente, la cual se sembró con pasto Prodigioso (Tripsacum laxum) durante la época lluviosa del 2011. Se utilizó una densidad de siembra de tres tallos de semilla vegetativa, colocadas las partes apical, media y basal en forma de cadena en el surco a una distancia entre hileras de $70 \mathrm{~cm}$. La primera cosecha se realizó 365 días después de la siembra, durante el segundo semestre del 2012. Se recogió una segunda cosecha y se uniformizó en mayo del 2013 para iniciar el experimento. A los quince días de edad, cuando el rebrote alcanzó una altura de $10 \mathrm{~cm}$, se aplicaron los tratamientos de fertilización.

El área de cultivo se dividió en 76 parcelas de $48 \mathrm{~m}^{2}$ para distribuir los tratamientos experimentales, se dejó dos metros de separación alrededor de cada una de ellas, dentro de cada uno de los cuatro bloques experimentales. El periodo experimental tuvo una duración de 120 días, desde la uniformización hasta la cosecha. Los niveles de nitrógeno $(\mathrm{N})$, fósforo $(\mathrm{P})$ y potasio $(\mathrm{K})$ centrales fueron: $225 \mathrm{~kg}$ de N/ha/año, $60 \mathrm{~kg} \mathrm{de} \mathrm{P}_{2} \mathrm{O}_{5} / \mathrm{ha} / \mathrm{año}$, y $150 \mathrm{~kg}$ de $\mathrm{K}_{2} \mathrm{O} /$ ha/año, formulándose el diseño de compuesto central con las dosis equivalentes según el diseño factorial rotable. Las cantidades empleadas fueron dosis equivalentes a 35, 112,5, 225, 337,5 y 414,2 kg/ha/año de nitrógeno $\left(\mathrm{N}_{2}\right) ; 9,55,30,60,90$ y $110,45 \mathrm{~kg} / \mathrm{ha} /$ año de fósforo $\left(\mathrm{P}_{2} \mathrm{O}_{5}\right)$ y $23,87,75,150,225$ y $276,13 \mathrm{~kg} / \mathrm{ha} /$ año de potasio

Cuadro 1. Descripción de las diferentes combinaciones de nitrógeno, fósforo y potasio $(\mathrm{N}, \mathrm{P}, \mathrm{K})$ utilizados como tratamientos y los valores de respuesta del contenido de materia seca, proteína cruda, fibra neutro detergente, extracto etéreo y cenizas de las diferentes partes del Tripsacum laxum. Estación Experimental Alfredo Volio Mata, Cartago, Costa Rica. 2013.

Table 1. Description of the different nitrogen, phosphorus, and potassium (N, P, K) combinations used as treatments and the obtained response values of dry matter content, crude protein, neutral detergent fiber, ether extract, and ash from the different parts of the Tripsacum laxum. Alfredo Volio Mata Experimental Farm. Cartago, Costa Rica. 2013.

\begin{tabular}{|c|c|c|c|c|c|c|c|c|c|c|c|c|c|c|c|c|c|c|}
\hline \multicolumn{3}{|c|}{ Tratamientos } & & \multicolumn{5}{|c|}{ Hoja } & \multicolumn{5}{|c|}{ Tallo } & \multicolumn{5}{|c|}{ Planta entera } \\
\hline $\mathbf{N}\left(\mathbf{N}_{2}\right)$ & $\begin{array}{c}\mathbf{P} \\
\left(\mathbf{P}_{2} \mathbf{O}_{5}\right)\end{array}$ & $\mathbf{K}$ & $\mathbf{n}$ & MS & PC & FND & 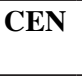 & $\mathbf{E E}$ & & 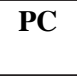 & FND & CEN & $\mathbf{E E}$ & MS & PC & FND & CEN & $E$ \\
\hline \multicolumn{3}{|c|}{ kg/ha/año } & & & & $\%$ & & & & & $\%$ & & & & & $\%$ & & \\
\hline 35,00 & 60,00 & , & 4 & 6 & 4 & 73,03 & & 3 & 1,38 & 0 & 66,14 & & 1,11 & 19,66 & 8 & 70,44 & & 1,46 \\
\hline , & & & 4 & 46 & +3 & 4 & & 1,80 & 1 & 42 & 1 & 4 & 8 & 17,37 &, 55 & & & ,60 \\
\hline 0 & & & 4 & & 4 & 0 & & 4 & $11, / 4$ & 6,28 & $0, / 0$ & 9 & 1,02 & 17,71 & 88 & & & ,28 \\
\hline 1. & & & 4 & 86 & 9 & 7 & 2,68 & 2,05 & וני, & 19 & oc & J & 1,26 & & 7 & & &, 75 \\
\hline, 50 & & & 4 & 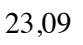 & 4 & 7 & & $J$ & & 8,55 & 0 & 1 & 0 & & 85 & & & 2,07 \\
\hline 22 & & & 4 & 7 & & & & 4 & & 7,79 & 0 & & 5 & & 31 & & & ,92 \\
\hline 22 & & & 4 & & & & & & & 7,14 & & & & & & & & 51 \\
\hline & & & 20 & & & & & & & & & & & & & & & 00 \\
\hline 225,00 & & 2 & 4 & 23,51 & 2 & 72 & 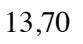 & 2,06 & דונים & 8,44 & 69, & 14,64 & 140 & 0 & 10,67 & & ; & 1,81 \\
\hline 22 & & & 4 & 24,64 & & 7 & & & 1 & 7,75 & 65 & 7 & 1,46 & 17 & 10,08 & & & 1,79 \\
\hline 337,5 & & & 4 & & & 71 & & 1,95 & 4 & 0 & 66 & & 1 , & & 44 & & & 1,78 \\
\hline 337,50 & & 25,00 & 4 & 313 & 07 & 71 & 30 & 2,31 & 11,46 & 1 & 63,20 & 12,51 & 1,63 & 16 & 12,92 & & 8 & 2,06 \\
\hline 33 & 00 & 75,00 & 4 & 22,81 & 15,83 & 70,22 & 13,03 & 2,54 & 12,18 & 10,30 & 63,80 & 14,35 & 1,83 & 17,01 & 13,75 & 31 & 13,52 & 2,28 \\
\hline 337,5 & 90,00 & 50 & 4 & 22,98 & ,97 & 69,15 & 13,62 & 1,89 & 12,98 & 10,39 & 66,38 & 14,12 & 1,68 & 17,16 & 11,37 & 1 & 1 & 1,81 \\
\hline 414,20 & 60,00 & 50,00 & 4 &, 64 & 32 & 70,82 & 2,45 & 1,98 & 12,15 & 9,48 & 63,02 & 12,36 & 1,59 & 17,38 & 11,87 & 67,29 & 12,42 & 1,84 \\
\hline
\end{tabular}

MS: materia seca, PC: proteína cruda, FND: fibra neutro detergente, CEN: cenizas totales, EE: extracto etéreo, como \% / MS: dry matter, PC: crude protein, FND: neutral detergent fiber, CEN: total ashes, EE: ether extract, as a \%. 
$\left(\mathrm{K}_{2} \mathrm{O}\right)$. La mezcla física se distribuyó de manera uniforme en cada parcela. El cultivo fue supervisado cada semana durante todo el periodo experimental, realizándose las labores culturales necesarias (control de malas hierbas, gusanos e insectos radicales y foliares, hongos y taltuzas o roedores) y un seguimiento visual de la evolución del crecimiento (altura, grosor y vigor de la planta).

La cosecha se realizó en cada parcela experimental, se tomaron cuatro muestras de material por tratamiento dentro de cada bloque. Las muestras fueron pesadas en verde y llevadas al laboratorio de bromatología de la estación experimental para la separación en hojas y tallos, así como los análisis químicos respectivos. Ambas fracciones se pesaron por separado y se secaron a $60{ }^{\circ} \mathrm{C}$ durante 48 horas hasta alcanzar un peso constante y pesadas nuevamente en seco. El contenido de materia seca (MS) se determinó en cada una de las 152 muestras experimentales, sometiéndolas a $105{ }^{\circ} \mathrm{C}$ mediante el procedimiento descrito por el AOAC (2002). A cada una de ellas se le determinó el contenido de extracto etéreo (EE) (AOAC, 2000), proteína cruda (PC) (Sosa-de-Pro, 1979), fibra en detergente neutro (FND), fibra en detergente ácido (FAD) y lignina mediante el procedimiento de Goering y Van-Soest (1970). La celulosa y hemicelulosa se obtuvieron por diferencia (Sosa-de-Pro, 1979).

\section{Diseño experimental}

El diseño experimental utilizado fue de bloques al azar con cuatro repeticiones y los tratamientos se dispusieron en un arreglo factorial de compuesto central y rotable $5^{3}$ (Cochran y Cox, 1957; Boschini, 2008). El modelo estadístico empleado fue el siguiente:

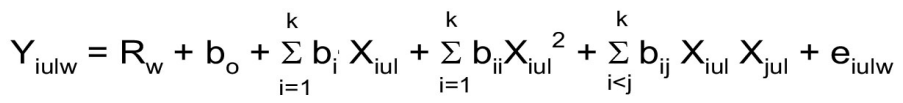

donde:

$\mathrm{Y}_{\text {iulw }}=$ observación l-ésima en w-ésimo bloque en el u-ésimo nivel del i-ésimo factor.

$\mathrm{R}_{\mathrm{w}}=$ bloque w-ésimo.

$\mathrm{b}_{\mathrm{o}}=$ valor de la intersección en la superficie de respuesta.

$\mathrm{b}_{\mathrm{i}}=$ pendiente lineal del i-ésimo factor.

$\mathrm{b}_{\mathrm{ii}}=$ pendiente cuadrática del i-ésimo factor.

$\mathrm{b}_{\mathrm{ij}}=$ pendiente de la interacción del i-ésimo factor x el j-ésimo factor.

$\mathrm{X}_{\mathrm{iul}}=$ observación l-ésima en el nivel u-ésimo del i-ésimo factor.

$\mathrm{X}_{\mathrm{jul}}=$ observación l-ésima en el nivel u-ésimo del j-ésimo factor.

$\mathrm{e}_{\text {iulw }}=$ error experimental en la observación l-ésima en el w-ésimo bloque en el u-ésimo nivel del i-ésimo factor.

Los resultados se analizaron con el PROC GLM del paquete estadístico SAS (1985) con el modelo descrito. Se realizó un ANOVA en cada uno de los cinco componentes bromatológicos estudiados, tanto en hojas como en tallos y planta entera, y se determinó los efectos lineales, cuadráticos y cúbicos de cada factor, así como las interacciones de primer orden entre los factores que resultaron significativos. Posteriormente, se escogieron los efectos significativos $(\mathrm{P}<0,01)$ y se introdujeron en una regresión polinomial múltiple con los tres factores (nitrógeno, fósforo y potasio) para cada componente bromatológico, tanto en hojas, tallos como en la planta entera. De cada regresión se extrajo las derivadas parciales correspondientes a cada factor, con el propósito de estimar individualmente el nivel de nitrógeno, fósforo y potasio $(\mathrm{kg} / \mathrm{ha} / \mathrm{año})$, donde se alcanza una respuesta porcentual mínima o máxima del componente bromatológico al cual se aplica. 


\section{Resultados}

Al explorar la respuesta bromatológica del Tripsacum laxum con diferentes niveles de N, P y K aplicados al suelo, los resultados obtenidos se detallan en en Cuadro 1, tanto en la planta entera como en la parte foliar y el tallo.

El contenido máximo de materia seca (MS) en planta entera de Tripsacum laxum obtenido fue de 15,85\%, cuando se aplicó una dosis de nitrógeno (N) de 265,64 kg/ha/año (Cuadro 2). Al fraccionar el pasto en hojas y tallo, se observó que una aplicación equivalente al 54\% del nitrógeno usado para la máxima producción en la planta entera (145 kg/ha/año), permitió alcanzar los contenidos más altos de materia seca en tallos (11,09\%), y con 110 $\mathrm{kg} / \mathrm{ha}$ (equivalente al $41 \%$ ) se alcanzó el máximo contenido de materia seca $(24,06 \%)$ en hojas.

El contenido de proteína cruda mostró un incremento lineal en la planta entera conforme se aportó mayor cantidad de nitrógeno; por su parte, las hojas alcanzaron un contenido máximo de 13,5\% cuando la dosis de nitrógeno aplicado llegó a $399 \mathrm{~kg} \mathrm{~N} / \mathrm{ha} / \mathrm{año}$. Las ecuaciones que predicen el comportamiento bromatológico de la planta entera y fraccionada se detallan en el Cuadro 3.

El porcentaje de fibra en detergente neutro (FND) en la planta entera varió entre 67 y $71 \%$ en el conjunto de tratamientos; en hojas, el rango fue de 69 a 73\% y, en el tallo, de 63 a 69\%. Se observó que al aumentar la aplicación de nitrógeno, la concentración de FND incrementó en la planta entera de manera lineal y en forma cuadrática en tallos y hojas, con un mínimo de 66\% en el tallo al aplicarse $81 \mathrm{~kg} \mathrm{~N} / \mathrm{ha} / \mathrm{año}$, y un máximo de 70,5\% en la hoja al aplicarse $304 \mathrm{~kg} \mathrm{~N} / \mathrm{ha} / \mathrm{año}$.

El efecto de la fertilización nitrogenada en el contenido de extracto etéreo (EE) fue cuadrático en la hoja, el tallo y, consecuentemente, en la planta entera. En la hoja la concentración de EE fue de 1,50\% sin fertilización nitrogenada, y alcanzó un máximo de 2,17\% con la aplicación de $275 \mathrm{~kg} \mathrm{~N} / \mathrm{ha} / \mathrm{año}$; en el tallo, se obtuvo un 0,99\% de EE con una dosis de $0 \mathrm{~kg} \mathrm{~N} / \mathrm{ha} /$ año, y se incrementó a un máximo de 1,66\% con $390 \mathrm{~kg} \mathrm{~N} / \mathrm{ha} / \mathrm{año}$. Al estimar un valor ponderado conjunto de hojas y tallos, el contenido de EE en la planta entera fue 1,30\% cuando el cultivo no recibió fertilización nitrogenada, aumentando la concentración de EE hasta alcanzar un valor pico de 1,94\% cuando recibió $302 \mathrm{~kg} \mathrm{~N} / \mathrm{ha} / \mathrm{año}$.

El contenido mineral expresado en forma de cenizas totales mostró un valor medio cercano al 13\%, observándose concentraciones más altas con cantidades de nitrógeno inferiores a $70 \mathrm{~kg} / \mathrm{ha} / \mathrm{año}(13,23 \%$ en hojas, $12,81 \%$ en tallos, y $13,46 \%$ en la planta completa).

Con la adición de fósforo, los contenidos de materia seca más altos se lograron con cantidades pequeñas. La fracción vegetal que requirió más cantidad de ese elemento fueron los tallos que necesitaron 26,97 kg/ha/año para lograr 12,19\%; mientras la planta entera y las hojas demandaron el 35 y el 65\% de la dosis anterior para alcanzar contenidos de materia seca máximos de 15,42 y $23,32 \%$, respectivamente.

El Tripsacum laxum como planta entera mostró niveles de proteína cruda de $10,82 \%$ cuando recibió $23,75 \mathrm{~kg}$ P/ha/año, en las hojas el contenido fue $12,56 \%$ y en tallos de $8,21 \%$, con cantidades de fósforo de 22,14 y 32,68 $\mathrm{kg} \mathrm{P} / \mathrm{ha} / \mathrm{año}$, respectivamente.

$\mathrm{Al}$ analizar la fracción fibrosa, la diferencia en la cantidad de fósforo aplicado para alcanzar las proporciones máximas de fibra en detergente neutro variaron en un rango corto; la planta entera demandó $21,97 \mathrm{~kg} / \mathrm{ha} / \mathrm{año}$, las hojas necesitaron 19,99 kg/ha/año y los tallos 18,28 kg/ha/año; esa diferencia de un kilogramo entre las partes de la planta y la planta entera, generaron valores de fibra en detergente neutro de $68,60 \%, 70,70 \%$ y $64,55 \%$, en el orden antes mencionado. El mismo patrón se observó para extracto etéreo, pero en este caso los máximos rendimientos se lograron con la misma cantidad de fósforo, tanto para planta entera como para sus partes $(27 \mathrm{~kg} \mathrm{P} / \mathrm{ha} / \mathrm{año})$.

El contenido máximo de cenizas en hojas y planta entera fue alrededor de $13 \%$ con una dosis media de $65 \mathrm{~kg}$ $\mathrm{P} / \mathrm{ha} / \mathrm{año}$, mientras que las cenizas contenidas en el tallo alcanzaron un valor similar con una dosis equivalente al $48 \%$ de lo requerido por las hojas. 
Cuadro 2. Valores de nitrógeno, fósforo y potasio $(\mathrm{N}, \mathrm{P}, \mathrm{K})$ que produjeron los contenidos máximos de materia seca, proteína cruda, fibra en detergente neutro, extracto etéreo y cenizas en el forraje Tripsacum laxum. Estación Experimental Alfredo Volio Mata, Cartago, Costa Rica. 2013.

Table 2. Values of nitrogen, phosphorus and potassium $(\mathrm{N}, \mathrm{P}, \mathrm{K})$ that produced the maximum content of dry matter, crude protein, neutral detergent fiber, ether extract, and ash in the forage Tripsacum laxum. Alfredo Volio Mata Experimental Farm. Cartago, Costa Rica. 2013.

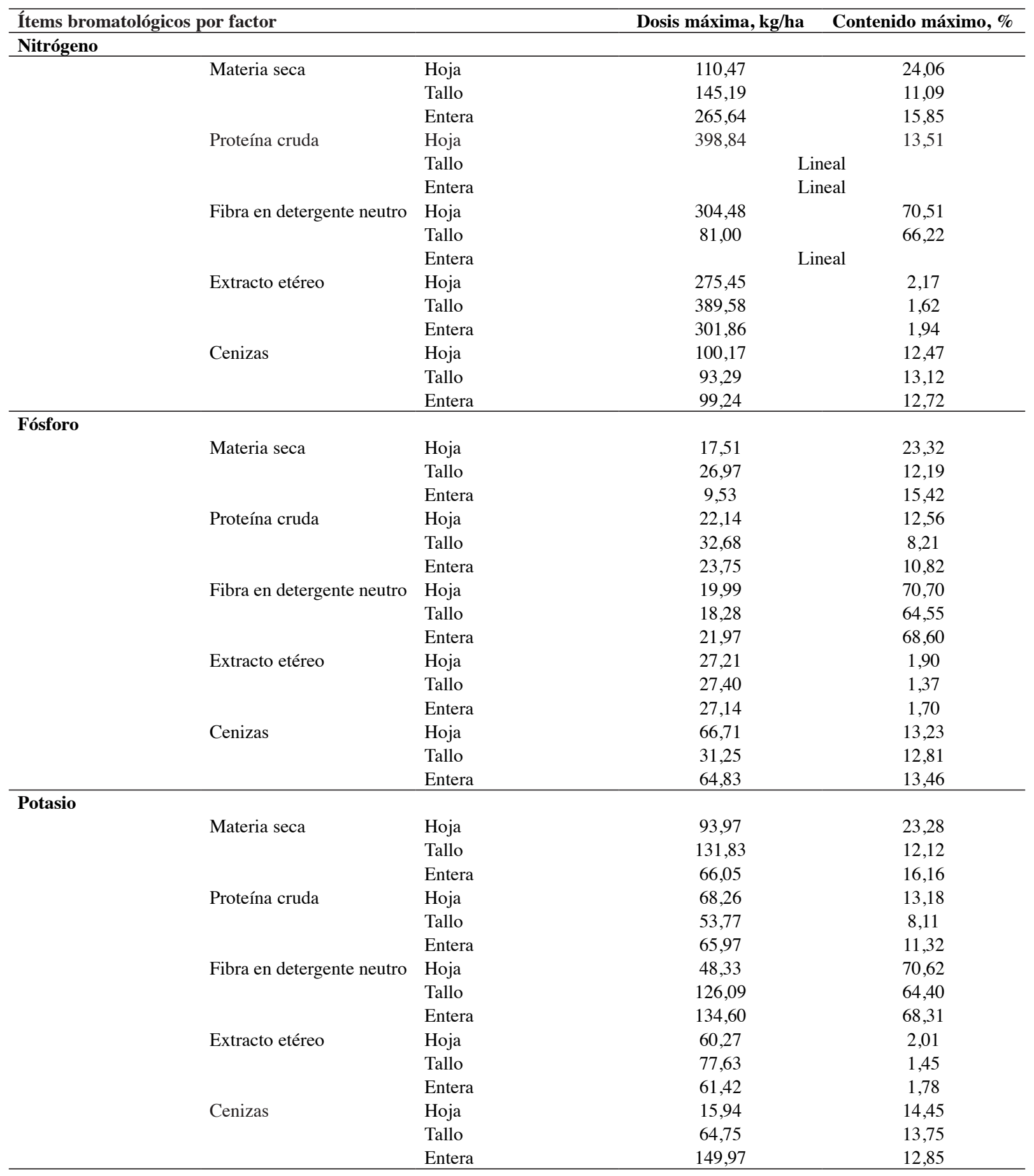


Cuadro 3. Predicción de calidad nutricional dependiendo de los niveles de nitrógeno, fósforo y potasio (N, P, K) aplicados al Tripsacum laxum. Estación Experimental Alfredo Volio Mata, Cartago. 2013.

Table 3. Prediction of nutritional quality depending on the different applied levels of nitrogen, phosphorus, and potassium (N, $\mathrm{P}, \mathrm{K})$ to the Tripsacum laxum. Alfredo Volio Mata Experimental Farm. Cartago, Costa Rica. 2013.

\begin{tabular}{|c|c|c|c|}
\hline \multicolumn{2}{|l|}{ Ítems bromatológicos por factor } & \multicolumn{2}{|l|}{ Ecuación de regresión } \\
\hline \multicolumn{4}{|l|}{ Nitrógeno } \\
\hline \multirow[t]{3}{*}{ Materia seca } & Hoja & $\mathrm{Y}=23,60450973+0,01631311 * \mathrm{~N}-0,00013819 * \mathrm{~N}^{2}+0,00000025 * \mathrm{~N}^{3}$ & $\mathrm{R}^{2}=0,71$ \\
\hline & Tallo & $\mathrm{Y}=11,15779642-0,00705253^{*} \mathrm{~N}+0,00005866 * \mathrm{~N}^{2}-0,00000009^{*} \mathrm{~N}^{3}$ & $\mathrm{R}^{2}=0,98$ \\
\hline & Entera & $\mathrm{Y}=20,53811998-0,03531959 * \mathrm{~N}+0,00006648 * \mathrm{~N}^{2}$ & $\mathrm{R}^{2}=0,92$ \\
\hline \multirow[t]{3}{*}{ Proteína cruda } & Hoja & $\mathrm{Y}=9,792602363+0,018640165^{*} \mathrm{~N}-0,000023368^{*} \mathrm{~N}^{2}$ & $\mathrm{R}^{2}=0,87$ \\
\hline & Tallo & $Y=6,612060879+0,00832565 * \mathrm{~N}$ & $\mathrm{R}^{2}=0,81$ \\
\hline & Entera & $\mathrm{Y}=9,050704505+0,008198254 * \mathrm{~N}$ & $\mathrm{R}^{2}=0,82$ \\
\hline \multirow[t]{3}{*}{ Fibra en detergente neutro } & Hoja & $\mathrm{Y}=73,39005553-0,01892624 * \mathrm{~N}+0,00003108 * \mathrm{~N}^{2}$ & $\mathrm{R}^{2}=0,86$ \\
\hline & Tallo & $\mathrm{Y}=66,04716803+0,00433047 * \mathrm{~N}-0,00002673 * \mathrm{~N}^{2}$ & $\mathrm{R}^{2}=0,91$ \\
\hline & Entera & $Y=70,44072516-0,00697707 * \mathrm{~N}$ & $\mathrm{R}^{2}=0,90$ \\
\hline \multirow[t]{3}{*}{ Extracto etéreo } & Hoja & $\mathrm{Y}=1,505752173+0,004808827 * \mathrm{~N}-0,000008729 * \mathrm{~N}^{2}$ & $\mathrm{R}^{2}=0,98$ \\
\hline & Tallo & $\mathrm{Y}=0,9907247899+0,0032241463 * \mathrm{~N}-0,000004138 * \mathrm{~N}^{2}$ & $\mathrm{R}^{2}=0,98$ \\
\hline & Entera & $\mathrm{Y}=1,305909753+0,004208537 * \mathrm{~N}-0,000006971 * \mathrm{~N}^{2}$ & $\mathrm{R}^{2}=0,98$ \\
\hline \multirow[t]{3}{*}{ Cenizas } & Hoja & $\mathrm{Y}=13,69642449-0,02505912 * \mathrm{~N}+0,00015246 * \mathrm{~N}^{2}-0,00000024 * \mathrm{~N}^{3}$ & $\mathrm{R}^{2}=0,88$ \\
\hline & Tallo & $\mathrm{Y}=13,92232255-0,01853234 * \mathrm{~N}+0,00012658 * \mathrm{~N}^{2}-0,00000022 * \mathrm{~N}^{3}$ & $\mathrm{R}^{2}=0,94$ \\
\hline & Entera & $\mathrm{Y}=13,78942467-0,02266308 * \mathrm{~N}+0,00014257 * \mathrm{~N}^{2}-0,00000023 * \mathrm{~N}^{3}$ & $\mathrm{R}^{2}=0,98$ \\
\hline \multicolumn{4}{|l|}{ Fósforo } \\
\hline \multirow[t]{3}{*}{ Materia seca } & Hoja & $\mathrm{Y}=22,77811735+0,0477393 * \mathrm{P}-0,00107856 * \mathrm{P}^{2}+0,00000714 * \mathrm{P}^{3}$ & $\mathrm{R}^{2}=0,69$ \\
\hline & Tallo & $\mathrm{Y}=11,78429835+0,04254162 * \mathrm{P}-0,00124817 * \mathrm{P}^{2}+0,0000085 * \mathrm{P}^{3}$ & $\mathrm{R}^{2}=0,99$ \\
\hline & Entera & $\mathrm{Y}=15,04588576+0,04185126 * \mathrm{P}-0,00022076 * \mathrm{P}^{2}$ & $\mathrm{R}^{2}=0,81$ \\
\hline \multirow[t]{3}{*}{ Proteína cruda } & Hoja & $14,9351456-0,17083825 * \mathrm{P}+0,00327335^{*} \mathrm{P}^{2}-0,00001809 * \mathrm{P}^{3}$ & $\mathrm{R}^{2}=0,73$ \\
\hline & Tallo & $8,08136317-0,019628261 * \mathrm{P}+0,000953157 * \mathrm{P}^{2}-0,00000713 * \mathrm{P}^{3}$ & $\mathrm{R}^{2}=0,42$ \\
\hline & Entera & $\mathrm{Y}=12,357138861-0,11361387 * \mathrm{P}+0,00239025 * \mathrm{P}^{2}-0,00001389^{*} \mathrm{P}^{3}$ & $\mathrm{R}^{2}=0,54$ \\
\hline \multirow[t]{3}{*}{ Fibra en detergente neutro } & Hoja & $\mathrm{Y}=68,63721277+0,15250704 * \mathrm{P}-0,00276787 * \mathrm{P}^{2}+0,00001498 * \mathrm{P}^{3}$ & $\mathrm{R}^{2}=0,94$ \\
\hline & Tallo & $\mathrm{Y}=62,93474645+0,11405182 * \mathrm{P}-0,00153207 * \mathrm{P}^{2}+0,00000649 * \mathrm{P}^{3}$ & $\mathrm{R}^{2}=0,81$ \\
\hline & Entera & $\mathrm{Y}=\quad 66,35831998+0,15325071 * \mathrm{P}-0,00263012 * \mathrm{P}^{2}+0,000013359 * \mathrm{P}^{3}$ & $\mathrm{R}^{2}=0,99$ \\
\hline \multirow{3}{*}{ Extracto etéreo } & Hoja & $\mathrm{Y}=2,417102995-0,03680836 * \mathrm{P}+0,000772012 * \mathrm{P}^{2}-0,000004288 * \mathrm{P}^{3}$ & $\mathrm{R}^{2}=0,97$ \\
\hline & Tallo & $\mathrm{Y}=1,762984541-0,027068911 * \mathrm{P}+0,000537556 * \mathrm{P}^{2}-0,000002872 * \mathrm{P}^{3}$ & $\mathrm{R}^{2}=0,99$ \\
\hline & Entera & $\mathrm{Y}=2,176674893-0,033567796 * \mathrm{P}+0,000694069^{*} \mathrm{P}^{2}-0,000003822 * \mathrm{P}^{3}$ & $\mathrm{R}^{2}=0,99$ \\
\hline \multirow[t]{3}{*}{ Cenizas } & Hoja & $\mathrm{Y}=11,51230862+0,05146131 * \mathrm{P}-0,00038569 * \mathrm{P}^{2}$ & $\mathrm{R}^{2}=0,94$ \\
\hline & Tallo & $\mathrm{Y}=12,84340135-0,04495246 * \mathrm{P}+0,00181752 * \mathrm{P}^{2}-0,00001318 * \mathrm{P}^{3}$ & $\mathrm{R}^{2}=0,95$ \\
\hline & Entera & $\mathrm{Y}=11,56985705+0,05832499 * \mathrm{P}-0,00044984 * \mathrm{P}^{2}$ & $\mathrm{R}^{2}=0,96$ \\
\hline \multicolumn{4}{|l|}{ Potasio } \\
\hline \multirow[t]{3}{*}{ Materia seca } & Hoja & $23,18032966-0,00336534 * \mathrm{~K}+0,00006335 * \mathrm{~K}^{2}-0,00000017 * \mathrm{~K}^{3}$ & $\mathrm{R}^{2}=0,33$ \\
\hline & Tallo & $\mathrm{Y}=11,20973313+0,01382869 * \mathrm{~K}-0,00005245 * \mathrm{~K}^{2}$ & $\mathrm{R}^{2}=0,91$ \\
\hline & Entera & $\mathrm{Y}=17,7838801-0,04687854 * \mathrm{~K}+0,00039796 * \mathrm{~K}^{2}-0,0000009 * \mathrm{~K}^{3}$ & $\mathrm{R}^{2}=0,70$ \\
\hline \multirow[t]{3}{*}{ Proteína cruda } & Hoja & $\mathrm{Y}=9,031630991+0,105231743 * \mathrm{~K}-0,000755049 * \mathrm{~K}^{2}+0,000001505 * \mathrm{~K}^{3}$ & $\mathrm{R}^{2}=0,73$ \\
\hline & Tallo & $\mathrm{Y}=6,415352117+0,043897433 * \mathrm{~K}-0,000255856 * \mathrm{~K}^{2}+0,000000456 * \mathrm{~K}^{3}$ & $\mathrm{R}^{2}=0,56$ \\
\hline & Entera & $\mathrm{Y}=8,044945999+0,082249907 * \mathrm{~K}-0,000567003 * \mathrm{~K}^{2}+0,000001108 * \mathrm{~K}^{3}$ & $\mathrm{R}^{2}=0,65$ \\
\hline \multirow[t]{3}{*}{ Fibra en detergente neutro } & Hoja & $\mathrm{Y}=\quad 68,50068029+0,06434424 * \mathrm{~K}-0,00047525 * \mathrm{~K}^{2}+0,00000105 * \mathrm{~K}^{3}$ & $\mathrm{R}^{2}=0,95$ \\
\hline & Tallo & $\mathrm{Y}=67,65724831-0,05158923 * \mathrm{~K}+0,00020458 * \mathrm{~K}^{2}$ & $\mathrm{R}^{2}=0,96$ \\
\hline & Entera & $\mathrm{Y}=\quad 70,62403106-0,0344007 * \mathrm{~K}+0,00012779 * \mathrm{~K}^{2}$ & $\mathrm{R}^{2}=0,88$ \\
\hline \multirow[t]{3}{*}{ Extracto etéreo } & Hoja & $\mathrm{Y}=1,357048638+0,016310763 * \mathrm{~K}-0,0001035542 * \mathrm{~K}^{2}+0,000000194 * \mathrm{~K}^{3}$ & $\mathrm{R}^{2}=0,99$ \\
\hline & Tallo & $\mathrm{Y}=1,065317228+0,00779258 * \mathrm{~K}-0,000041086 * \mathrm{~K}^{2}+0,000000063 * \mathrm{~K}^{3}$ & $\mathrm{R}^{2}=0,95$ \\
\hline & Entera & $\mathrm{Y}=1,2396431+0,013312359 * \mathrm{~K}-0,000081574 * \mathrm{~K}^{2}+0,000000148 * \mathrm{~K}^{3}$ & $\mathrm{R}^{2}=0,99$ \\
\hline \multirow[t]{3}{*}{ Cenizas } & Hoja & $\mathrm{Y}=14,4678132-0,002320152 * \mathrm{~K}+0,00007278 * \mathrm{~K}^{2}$ & $\mathrm{R}^{2}=0,79$ \\
\hline & Tallo & $\mathrm{Y}=13,29042002+0,01883305 * \mathrm{~K}-0,00022256 * \mathrm{~K}^{2}+0,00000062 * \mathrm{~K}^{3}$ & $\mathrm{R}^{2}=0,99$ \\
\hline & Entera & $\mathrm{Y}=14,35159641-0,02004836 * \mathrm{~K}+0,00006684 * \mathrm{~K}^{2}$ & $\mathrm{R}^{2}=0,84$ \\
\hline
\end{tabular}


Las cantidades de fósforo que se necesitaron para obtener la máxima concentración de materia seca, proteína cruda, fibra en detergente neutro y extracto etéreo, tanto en las hojas como en el tallo, estuvieron en un rango de 10 a $33 \mathrm{~kg} \mathrm{P} / \mathrm{ha} / \mathrm{año.}$

La fertilización con potasio $(\mathrm{K})$ mostró que el contenido de materia seca en las hojas alcanzó un valor máximo de $23,28 \%$ con una dosis de $93,97 \mathrm{~kg} \mathrm{~K} / \mathrm{ha} / \mathrm{año}$, equivalente a un $75 \%$ de lo que requirieron los tallos para lograr un contenido pico de $12,12 \%$. No obstante, al observar la reacción de la planta entera a la fertilización potásica, el requerimiento de potasio se dio a un nivel menor que lo demandado por las hojas y tallos por separado. En la planta entera un nivel de potasio requerido para alcanzar un contenido máximo de 16,16\% fue de $66,05 \mathrm{~kg} \mathrm{~K} / \mathrm{ha} / \mathrm{año}$. Por su parte, la dosis de potasio que se necesitó para generar los mejores resultados de proteína cruda $(11,32 \%$ para la planta entera, $13,18 \%$ en hojas y $8,11 \%$ en tallos) no sobrepasaron los $68,26 \mathrm{~kg} \mathrm{~K} / \mathrm{ha} / \mathrm{año}$, cantidad muy parecida a la que dio el valor más alto de materia seca en la planta entera.

En la fracción fibrosa en detergente neutro (FND), se observó que una dosis de potasio de $48 \mathrm{~kg} / \mathrm{ha} / \mathrm{años}$ permitió alcanzar un máximo de $70 \%$ en las hojas, $126 \mathrm{~kg} / \mathrm{ha} /$ año para que el tallo mostrara un máximo de $64 \%$ y $140 \mathrm{~kg} / \mathrm{ha} /$ año para que la planta entera tuviera un $68,31 \%$ de fibra en detergente neutro; no obstante, estas cantidades exceden en un $250 \%$ la dosis que requirieron hojas.

El efecto del potasio sobre la concentración pico del extracto etéreo se manifestó entre 60 a $77 \mathrm{~kg} \mathrm{~K} / \mathrm{ha} / \mathrm{año}$, para hoja y tallo, respectivamente; con un valor intermedio para la planta entera. Contrario a esta respuesta, se observaron marcadas diferencias en la cantidad requerida de potasio para alcanzar las máximas concentración de cenizas en hojas y tallos (13-14\%).

\section{Discusión}

La producción de materia seca es el parámetro sobre el cual se centra la composición nutricional de un material, en el caso del Tripsacum laxum el valor obtenido fue de 15,85\% cuando la dosis de N suministrada fue de 265,64 $\mathrm{kg} / \mathrm{ha} / \mathrm{año}$. Este dato es ligeramente mayor a los resultados de 15,49\% obtenidos en un estudio que evaluó la misma especie a una edad de cosecha de 365 días (Vargas-Rodríguez, 2009). Esto indica que la fertilización nitrogenada en el pasto Prodigioso favoreció el crecimiento y, que a edades más cortas, se logró un adecuado contenido de MS; sin embargo, resultó contrario a lo expuesto en otros reportes, donde se menciona que conforme aumenta la edad de la planta aumenta el contenido de materia seca (Amador y Boschini, 2000).

Los resultados observados en cuanto a los rendimientos porcentuales de MS en las fracciones del T. laxum permiten considerar la regulación de la dosis de nitrógeno de acuerdo con el objetivo que se quiere con un plan de fertilización, y así maximizar la calidad y rentabilidad. Por ejemplo, para maíz se indica que con dosis de $150 \mathrm{~kg}$ N/ha/año se logra la producción económica máxima de este cultivo, y con $200 \mathrm{~kg} \mathrm{~N} / \mathrm{ha} /$ año aumenta la calidad del mismo cuando se usa para ensilaje (Cox et al., 1993).

El comportamiento lineal observado en el contenido de proteína cruda que mostró el pasto Prodigioso, mostró el efecto que tiene el nitrógeno sobre la estructura de la proteína y la elongación de los tallos (Frink et al., 1999). Esta conducta se asemeja a los datos obtenidos en maíz, donde al evaluar dosis ascendentes de nitrógeno, el contenido proteico se incrementó más del 100\% al aplicar $400 \mathrm{~kg} / \mathrm{ha}$ de nitrógeno (Soto et al., 2004).

Los porcentajes de la fracción fibrosa en el T. laxum observados en este estudio fueron muy similares a un reporte previo donde se obtuvieron valores de 68,58\% de FND (Vargas-Rodríguez, 2009), pero fueron superiores a otro trabajo realizado con esta misma especie donde el valor de FDN fue de 63,9\% (Mlay et al., 2006). Ambos trabajos utilizaron $T$. laxum de edades avanzadas (> 150 días), lo que hace suponer que es un material forrajero que mantiene muy constante los contenidos fibrosos al pasar el tiempo. Al comparar el pasto Prodigioso con otros materiales, los valores de FND obtenidos en este estudio resultaron menores a los valores de 73,36\% de FND 
reportados en el sorgo negro forrajero (Sorghum almum) (Vargas-Rodríguez, 2005), cosechado a setenta días de edad y fertilizado con $95 \mathrm{~kg} \mathrm{~N} / \mathrm{ha} /$ año. Incluso, fueron valores menores a lo reportado en estrella africana (Cynodon nlemfluensis) de 72,3\%, a una edad de cosecha de 25 días (Sánchez y Soto, 1999).

En cuanto al efecto de la fertilización nitrogenada en el contenido de EE, el rango de 1,66 a 2,17\% obtenidos en las fracciones y la planta entera se encuentran en un nivel aceptable para usarse en la alimentación de vacas lecheras, debido a que contenidos superiores al 5\% pueden afectar la digestión de la fibra y el consumo de materia seca (Tobía y Villalobos, 2004).

Cuando se confrontan los resultados de este estudio, en términos del contenido de cenizas, con datos obtenidos en maíz cosechado a una edad parecida (Boschini-Figueroa y Elizondo-Salazar, 2004), los tallos y la planta entera de maíz (6,74\% y 8,67\%, respectivamente) no superaron los valores que mostró el T. laxum; sin embargo, el contenido de EE en las hojas de ambas especies mostraron semejanza $(13,63 \%)$.

La suplementación adecuada de fósforo evita que aspectos como el desarrollo foliar se vea afectado, la deficiencia de este elemento en maíz ha sido asociada a una disminución en el tamaño de las hojas y el área foliar, ocasionado por una reducción en la división celular (Assuero et al., 2004). Los resultados en T. laxum indican que, incrementos significativos en el contenido de materia seca se pueden alcanzar con pequeñas dosis del elemento P. Al comparar la concentración de proteína cruda del Prodigioso utilizado en este estudio, con información perteneciente a materiales del mismo género, pero de diferente especie como el Trypsacum dactyloides, se observó que, el contenido proteico de las hojas es inferior, mientras que en los tallos son muy similares según lo reportado en un trabajo donde se observó que el $T$. dactyloides mostró 18,6\% de proteína cruda en hojas y 8,2\% en tallos (Bidlack et al., 1999). Sin embargo, los datos de planta entera resultaron ligeramente superiores al 9,1\% de proteína cruda que se menciona en otro trabajo también realizado con T. dactyloides (Horner et al., 1985).

En relación a la fracción fibrosa, el material experimental presentó una producción de fibra alta cuando la dosis de $\mathrm{P}$ aplicada estuvo entre 18 y $20 \mathrm{~kg} / \mathrm{ha} / \mathrm{año}$, cantidades que fueron inferiores a las utilizadas en cebada, donde la aplicación de dosis de fósforo de $60 \mathrm{~kg} /$ ha propiciaron los niveles más altos de fibra en detergente neutro en ese cultivo (Mehrvarz y Chaichi, 2008). Sin embargo, en ese mismo trabajo no se encontraron diferencias importantes cuando aplicaron $30 \mathrm{~kg} \mathrm{P} / \mathrm{ha} /$ año, esta última cantidad se asemeja más a lo obtenido en este trabajo.

Las cantidades de fósforo requeridas para maximizar las características nutricionales del T. laxum están en un rango de 10 a $33 \mathrm{~kg} \mathrm{P} / \mathrm{ha} /$ año, niveles relativamente bajos con respecto a lo que se menciona para forrajes de corte como el Sorghum bicolor (L.) Moench, donde se obtienen óptimos resultados con cantidades de $80 \mathrm{~kg}$ P/ha/ año (Patel, 2006), esto se puede atribuir a la presencia residual de $\mathrm{P}$ en el suelo producto de fertilizaciones previas (García y Ciampitti, 2009); a pesar que el fósforo presenta problemas de fijación en suelos de origen volcánico, manteniéndose poco disponible para la planta (Cabalceta, 1999).

Las dosis de potasio obtenidas en este experimento para alcanzar los máximos contenidos de materia seca y proteína cruda en la planta entera del T. laxum, permitieron superar la concentración de materia seca reportada en el sorgo negro con edades de 56 y 70 días de rebrote, pero es similar cuando se cosechó a 84 días (Elizondo-Salazar, 2004). Sin embargo, los contenidos de proteína cruda reportados en el sorgo negro a los 56, 70 y 80 días de rebrote fueron de $15,02 \%, 12,76 \%$ y $12,47 \%$, respectivamente, los cuales superaron a los obtenidos en este trabajo.

El contenido de fibra en detergente neutro (FND) que se observó cuando el potasio fue suministrado al cultivo de Prodigioso, concuerda con un estudio realizado en maíz sembrado a 30, 50 y $70 \mathrm{~cm}$ de distancia entre plantas, donde se reportaron valores contenidos de FND en la hoja de 70,85\%, 71,57\% y 69,20\%, en el orden de las distancias de siembra mencionado previamente, con una fertilización de $44 \mathrm{~kg} \mathrm{~K} / \mathrm{ha} / \mathrm{año} \mathrm{(Elizondo} \mathrm{y} \mathrm{Boschini,} \mathrm{2001).}$

Los contenidos máximos de cenizas fueron similares a lo reportado para Prodigioso con una edad de cosecha de 365 días, donde el promedio fue de 13,42\% (Vargas, 2009); pero fueron más altos que los valores de 12,87\%, 12,35\%, 12,27\% (Elizondo y Boschini, 2001) y 8,67\% (Boschini-Figueroa y Elizondo-Salazar, 2004) reportados en maíz. 
Los programas de fertilización se centran en combinar elementos para potenciar la respuesta del cultivo, evitando las interacciones entre los nutrientes que pueden afectar la absorción de un elemento con respecto a otro (Fageria, 2001). En el Cuadro 1 se pueden observar los resultados de la composición nutricional del T. laxum cuando se combinaron el nitrógeno, fósforo y potasio. El forraje utilizado mostró las mejores características nutricionales cuando las cantidades combinadas de nitrógeno, fósforo y potasio fueron $337,50 \mathrm{~kg} / \mathrm{ha} / \mathrm{año}, 90 \mathrm{~kg} / \mathrm{ha} / \mathrm{año} \mathrm{y} 75 \mathrm{~kg} /$ ha/año respectivamente. El valor de materia seca obtenido con esta mezcla se ubica entre los más altos de todos los tratamientos; pero su aporte se enfoca en el contenido proteico que fue el más alto, así como el extracto etéreo y convenientemente el contenido de fibra en detergente neutro fue el segundo más bajo de todos los tratamientos. La desventaja radica en la proporción de cenizas que fue una de las más bajas en este estudio.

Dependiendo del contenido bromatológico que se quiera favorecer, ya sea aumentándolo como en el caso de la proteína o disminuyéndolo en el caso de las fibras, los niveles óptimos de N, P y K requieren diferentes dosis de fertilizante, por ejemplo, si se desea mejorar el contenido de materia seca en la hoja una aplicación de $110 \mathrm{~kg} / \mathrm{ha} /$ año de N permite obtener un 24\% de MS, siendo suficiente alrededor de $17 \mathrm{~kg} / \mathrm{ha} / \mathrm{año} \mathrm{de} \mathrm{P}$ y $94 \mathrm{~kg} / \mathrm{ha} / \mathrm{año}$ de K. Sin embargo, para lograr hasta un $14 \%$ en el contenido de proteína cruda de las hojas se requiere $398 \mathrm{~kg}$ de N/ha/ año, con un efecto lineal sin tope de acumulación en el tallo. Con niveles de $22 \mathrm{~kg} / \mathrm{ha} /$ año de $\mathrm{P}$ se logra acumular un $13 \%$ de proteína en la hoja y solamente se necesitan $68 \mathrm{~kg} /$ ha/año de $\mathrm{P}_{2} \mathrm{O}_{5}$ para un máximo similar de proteína.

Otro de los propósitos de la aplicación de fertilizantes en forrajes con frecuencias de corta es optimizar la producción de biomasa que además proporcione la más alta calidad nutricional posible. Si la intención es aumentar el contenido de materia seca, la mejor combinación se obtuvo con 35, 60 y $150 \mathrm{~kg} / \mathrm{ha} /$ año de nitrógeno, fósforo y potasio, respectivamente. El porcentaje de materia seca logrado con esa combinación fue de 19,66\%, pero mostró el nivel más bajo de proteína $(8,98 \%)$ y el segundo más alto en fibra $(70,44 \%)$. El porcentaje de materia seca y la proporción de proteína están correlacionados negativamente, mientras que la fracción fibrosa puede aumentar conforme aumenta el contenido de materia seca (Juárez-Hernández y Bolaños-Aguilar, 2007). Con una cantidad más alta de potasio, aumentó el contenido de FND y disminuyó el porcentaje de proteína cruda, situación que se respalda con los resultados de pruebas realizadas en alfalfa, donde se observó una disminución en la concentración de la fracción fibrosa cuando se redujo la cantidad de potasio (Lissbran et al., 2009).

\section{Conclusiones}

En las hojas del pasto Prodigioso se logró el mayor contenido de materia seca con dos posibles combinaciones, 414,20, 60 y $150 \mathrm{~kg} / \mathrm{ha} /$ año de NPK, o con 225, 110,45 y $150 \mathrm{~kg} / \mathrm{ha} / \mathrm{año}$ de N, P, K, respectivamente, mientras que el mejor contenido de proteína cruda se logró con $337 \mathrm{~kg}$ de N/ha/año, $90 \mathrm{~kg}$ de P/ha/año, y $75 \mathrm{~kg}$ de K/ha/año, que fue la misma mezcla que dio los mejores resultados en planta entera.

La mezcla de nitrógeno, fósforo y potasio que favoreció las características nutricionales de los tallos fue la de $337,50,90$ y $225 \mathrm{~kg} / \mathrm{ha} / \mathrm{año}$, respectivamente. Esta combinación les permitió a los tallos tener 12,98\% de materia seca, 10,39\% de proteína cruda que fueron los más altos de todos los tratamientos, niveles intermedios de fibra en detergente neutro $(66,38 \%)$ y cenizas $(14,12 \%)$, y el segundo porcentaje más alto de extracto etéreo $(1,68 \%)$.

\section{Literatura citada}

Amador, A.L., y C. Boschini. 2000. Calidad nutricional de la planta de sorgo negro forrajero (Sorghum almum) para la alimentación animal. Agron. Mesoam. 11(2):79-84. 
AOAC (Association of Official Analytical Chemistry). 2000. Official methods of analysis. 17 $7^{\text {th }}$ ed. AOAC Int., Gaithersburg, MD, USA.

AOAC (Association of Official Analytical Chemistry). 2002. Official methods of analysis. $19^{\text {th }}$ ed. AOAC Int., WA. USA.

Assuero, S.G., A. Mollier, and S. Pellerin. 2004. The decrease in growth of phosphorus-deficient maize leaves is related to lower cell production. Plant Cell Environ. 27:887-895. doi:10.1111/j.1365-3040.2004.01194.x

Bednarz, C.W., and D.M. Oosterhuis. 1999. Physiological changes associated with potassium deficiency in cotton. J. Plant Nutr. 22:303-313. doi:10.1080/01904169909365628

Bertsch, F. 1998. La fertilidad de los suelos y su manejo. Asociación Costarricense de la Ciencia del Suelo, San José, CRC.

Bidlack, J.E., J.E. Vaughan, and C.L. Dewald. 1999. Forage quality of 10 Eastern gamagrass (Trypsacum dactyloides (L.) L.) genotypes. J. Range Manag. 52:661-665. doi:10.2307/4003638

Boschini, C. 2008. Diseños experimentales especiales en la investigación con ganado lechero. Serie Agrotecnología. vol 6. Editorial Universidad de Costa Rica, CRC.

Boschini-Figueroa, C., y J.A. Elizondo-Salazar. 2004. Desarrollo productivo y cualitativo de maíz híbrido para ensilaje. Agron. Mesoam. 15:31-37. doi:10.15517/am.v15i1.11926

Cabalceta, G. 1999. Fertilización y nutrición de forrajes de altura. En: Ministerio de Agricultura y Ganandería, editor, XI Congreso Agronómico Nacional/ III Congreso de Suelos. Editorial Universidad de Costa Rica, San José, CRC. p. 239-254.

Cástino, E.G.. 2007. Pasturas: Estimación y modalidades de uso de fertilizantes en el mercado de pasturas. Fertilizar 7:10-17.

Chen, C.P. 1992. Tripsacum andersonii J.R. Gray. In: L. 't Mannetje, and R.M. Jones, editors, Plant resources of South-East Asia. No. 4. Forages. Centre for Agricultural Publishing and Documentation (PUDOC), Wageningen, HOL. p. $228-230$.

Cherney, D.J.R., J.H. Cherney, and A.N. Pell. 1994. Inorganic nitrogen supply effects on alfalfa forage quality. J. Dairy Sci. 77:230-236. doi:10.3168/jds.S0022-0302(94)76945-9

Cochran, W.G., and G.M. Cox. 1957. Experimental designs. $2^{\text {nd }}$ ed. John Wiley \& Sons, NY, USA.

Cox, W.J., S. Kalonge, D. Cherney, and W.S. Reid. 1993. Growth, yield and quality of forage maize under different nitrogen management practices. J. Agron. 85:341-347. doi:10.2134/agronj1993.00021962008500020033x

Elizondo, J., y C. Boschini. 2001. Efecto de la densidad de siembra sobre el rendimiento y calidad del forraje de maíz. Agron. Mesoam. 12:181-187.

Elizondo-Salazar, J.A. 2004. Consumo de sorgo negro forrajero (Sorghum almum) en cabras. Agron. Mesoam. 15:77-80. doi: 10.15517/am.v15i1.11932

Fageria, V.D. 2001. Nutrient interactions in crop plants. J. Plant Nutr. 24:1269-1290. doi:10.1081/PLN-100106981

Frink, C.R., P.E. Waggoner, and J.H. Ausubel. 1999. Nitrogen fertilizer: retrospect and prospect. Proc. Acad. Sci. 96:1175-1180. doi:10.1073/pnas.96.4.1175

García, F., e I. Ciampitti. 2009. Fertilización de forrajeras: algunas consideraciones para 2009. Informaciones Agronómicas 41:25.

Goering, H.K., and P.J. Van-Soest. 1970. Forage fiber analyses (apparatus, reagents, procedures, and some applications). Agricultural Handbook No. 379. USDA, WA, USA.

Horner, J.L., L.J. Bush, and G.D. Adams. 1985. Comparative nutritional value of Eastern Gamagrass and Alfalfa hay for dairy cows. J. Dairy Sci. 68:2615-2620. doi:10.3168/jds.S0022-0302(85)81145-0

IMN (Instituto Meteorológico Nacional). 2010. Información climatológica de la zona de Ochomogo, Cartago. IMN, San José, CRC. 
Juárez-Hernández, J., y E.D. Bolaños-Aguilar. 2007. Las curvas de dilución de la proteína como alternativa para la evaluación de pastos tropicales. Universidad y Ciencia 23:81-90.

Leblanc, O., D. Grimanelli, D. Gonzalez-de-Leon, and Y. Savidan. 1995. Detection of the apomictic mode of reproduction in maize-tripsacum hybrids using maize RFLP markers. Theor. Appl. Genet. 90:1198-1203. doi:10.1007/BF00222943

Lissbrant, S., S. Stratton, S.M. Cunningham, S.M. Brouder, and J.J. Volenec. 2009. Impact of long-term phosphorus and potassium fertilization on alfalfa nutritive value-yield relationships. Crop Sci. 49:1116-1124. doi:10.2135/cropsci2008.06.0333

Mehrvarz, S., and M.R. Chaichi. 2008. Effects of phosphate solubilizing microorganisms and phosphorus chemical fertilizer on yield and yield components of barely (Hordeum vulgare L.). American-Eurasian. J. Agric. Environ. Sci. 3:822-828.

Mlay, P.S., A. Pereka, E. Chikula-Phiri, S. Balthazary, J. Igusti, T. Hvelplund, M. Riis-Weisbjerg, and J. Madsen. 2006. Feed value of selected tropical grasses, legumes and concentrates. Veterinarski Arh. 76:53-63.

Patel, P.C. 2006. Effect of phosphorus and potassium application on forage yield, quality, nutrient uptake and persistence of lucerne (Medicago sativa L.). In: International Union of Soil Science, editor, The $18^{\text {th }}$ World Congress of Soil Science. International Union of Soil Science, Philadelphia, PA, USA. p. 165-173.

Quintero, C.E., N.G. Boschetti, y R.A. Benavidez. 1997. Efecto residual y refertilización fosfatada de pasturas implantadas en Entre Ríos (Argentina). Ciencia del suelo 15:1-5.

Romero, C., y O. Márquez. 2002. Efecto de la fertilización fosforada en pasto Brachiaria humidicola sobre la producción láctea de vacas de doble propósito. Rev. Cient. 12:578-580.

Sánchez, J.M., y H. Soto. 1999. Calidad nutricional de los forrajes de una zona con niveles medios de producción de leche, en el trópico húmedo del norte de Costa Rica. Agron. Costarricense 23(2):165-171.

SAS. 1985. SAS user's guide: Statistics. SAS Institute Inc. Cary, NC. USA.

Soil Survey Staff, editor. 2014. Keys to soil taxonomy. 12th ed. USDA-Natural Resources Conservation Service, WA, USA.

Sosa-de-Pro, E. 1979. Manual de procedimientos analíticos para alimentos de consumo animal. Universidad Autónoma de Chapingo, MEX.

Soto, P., E. Jahn, y S. Arredondo. 2004. Mejoramiento del porcentaje de proteína en maíz para ensilaje con un aumento y parcialización de la fertilización nitrogenada. Agric.Téc. 64:156-162. doi:10.4067/S0365-28072004000200004

Suyama, H., S.E. Benes, P.H. Robinson, G. Getachew, S.R. Grattan, and C.M. Grieve. 2007. Biomass yield and nutritional quality of forage species under long-term irrigation with saline-sodic drainage water: Field evaluation. Anim. Feed Sci. Technol. 135:329-345. doi:10.1016/j.anifeedsci.2006.08.010

Tobía, C., y E. Villalobos. 2004. Producción y valor nutricional de forrajes de soya en condiciones tropicales adversas. Agron. Costarricense 28(1):17-25.

Torres, M., y J. Lemos 2009. Influencia de la fertilización con nitrógeno y azufre sobre la producción de forraje de raigrás anual. Informaciones Agronómicas 41:22-24.

Vargas-Rodríguez, C.F. 2005. Valoración nutricional y degradabilidad ruminal de genotipos de sorgo forrajero (Sorghum sp.). Agron. Mesoam. 16:215-223. doi:10.15517/am16i2.11874

Vargas-Rodríguez, C.F. 2009. Consumo y calidad del forraje Trypsacum laxum de un año de edad en cabras. Agron. Mesoam. 20:391-398. doi:10.15517/am.v20i2.4955

Vásquez, A. 1982. Estudio detallado de los suelos de la Estación Experimental de Ganado Lechero El Alto. Universidad de Costa Rica, CRC.

Veneciano, J.H., y K.L. Frigerio. 2008. Efecto de la fertilización nitrogenada en digitaría (Digitaria eriantha Steudel). Informaciones Agronómicas 37:12-16. 